\title{
NAWDEX
}

NATIONAL WATER DATA EXCHANGE

\section{DIRECTORY OF ASSISTANCE CENTERS OF THE NATIONAL WATER DATA EXCHANGE (NAWDEX)}

\section{U.S. GEOLOGICAL SURVEY}

Open-File Report 95-144 


\title{
DIRECTORY OF ASSISTANCE CENTERS OF THE NATIONAL WATER DATA EXCHANGE (NAWDEX)
}

\section{By Cassandra D. Blackwell}

\author{
U.S. Geological Survey \\ Open-File Report 95-144 \\ (Supersedes Open-File Report 93-76)
}

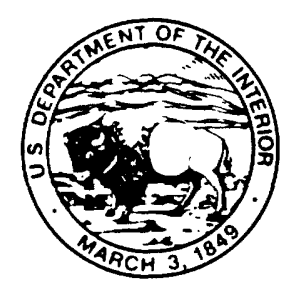

Reston, Virginia

1994 


\section{U.S. DEPARTMENT OF THE INTERIOR BRUCE BABBITT, Secretary \\ U.S. GEOLOGICAL SURVEY \\ Gordon P. Eaton, Director}

For additional information write to:

Chief Hydrologist U.S. Geological Survey 421 National Center Reston, Virginia 22092
Copies of this report can be purchased from:

U.S. Geological Survey

Earth Science Information Center

Open-File Reports Section Box 25286, MS 517

Denver Federal Center Denver, Colorado 80225 


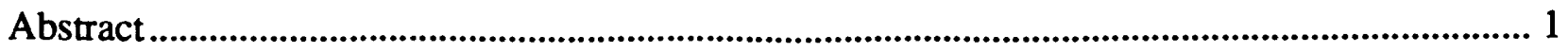

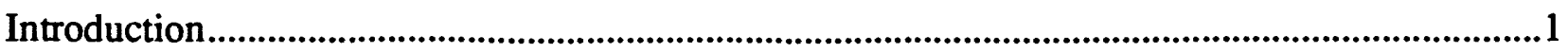

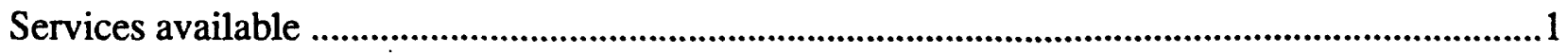

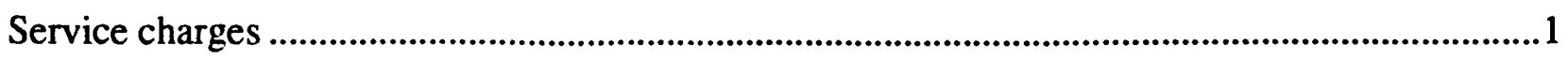

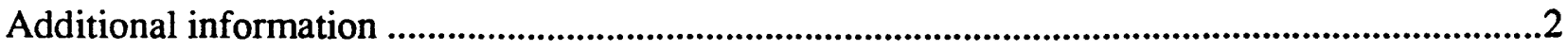

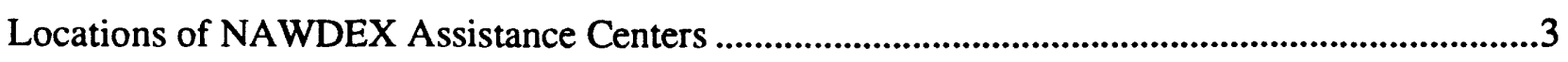

Alabama

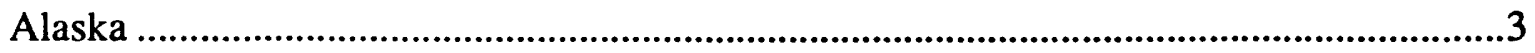

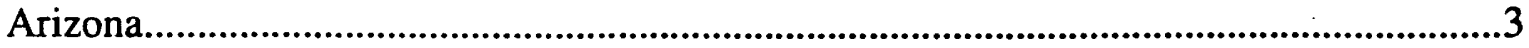

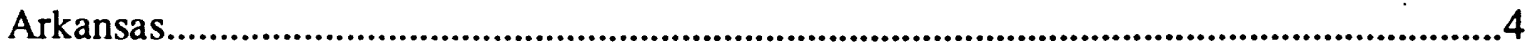

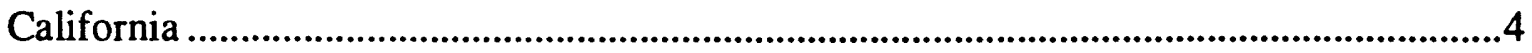

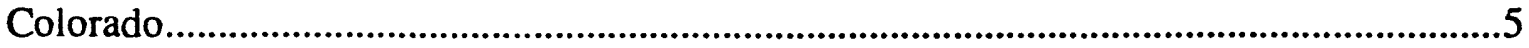

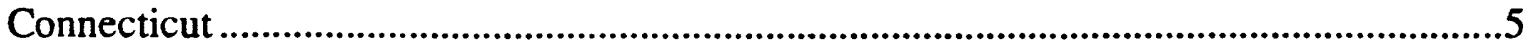

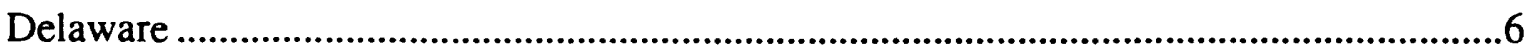

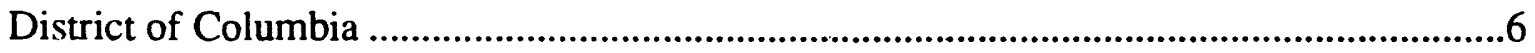

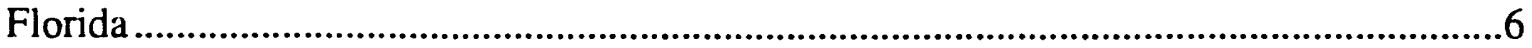

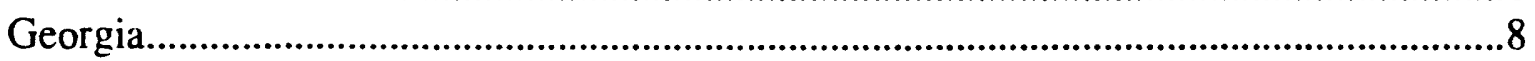

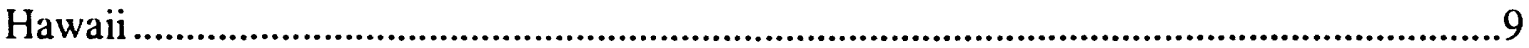

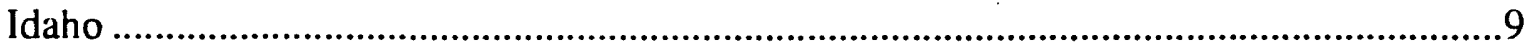

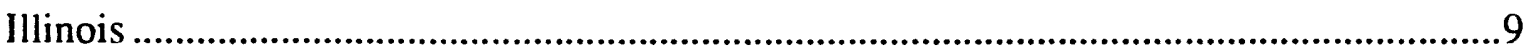

Indiana

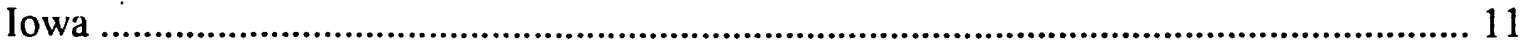

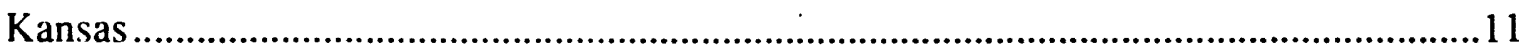

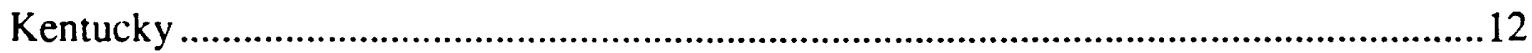

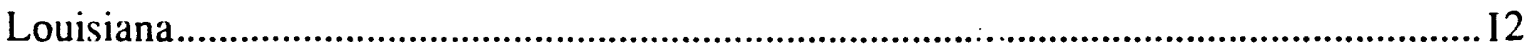

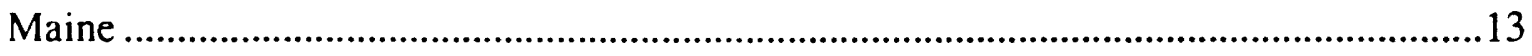

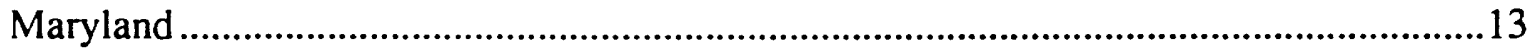

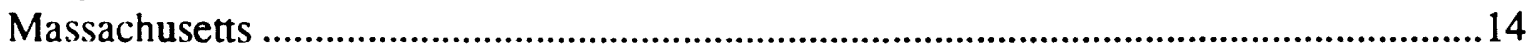

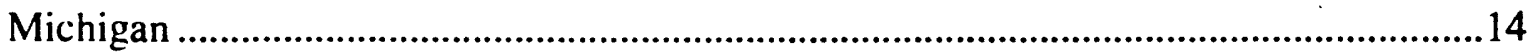

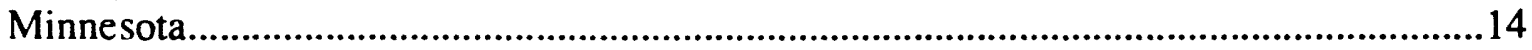

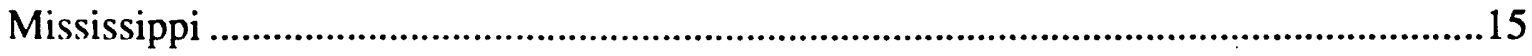

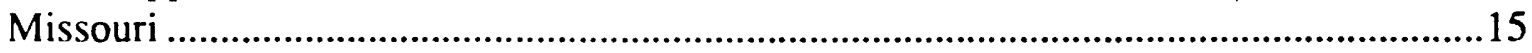

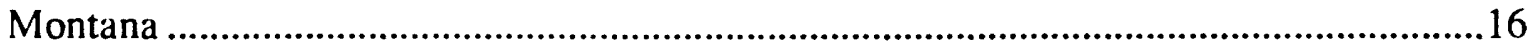

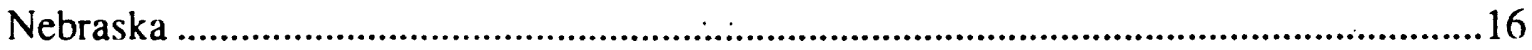

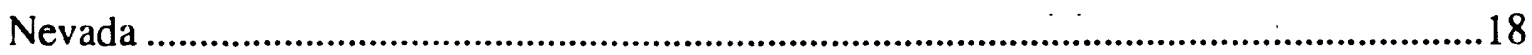

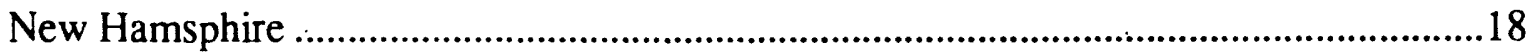




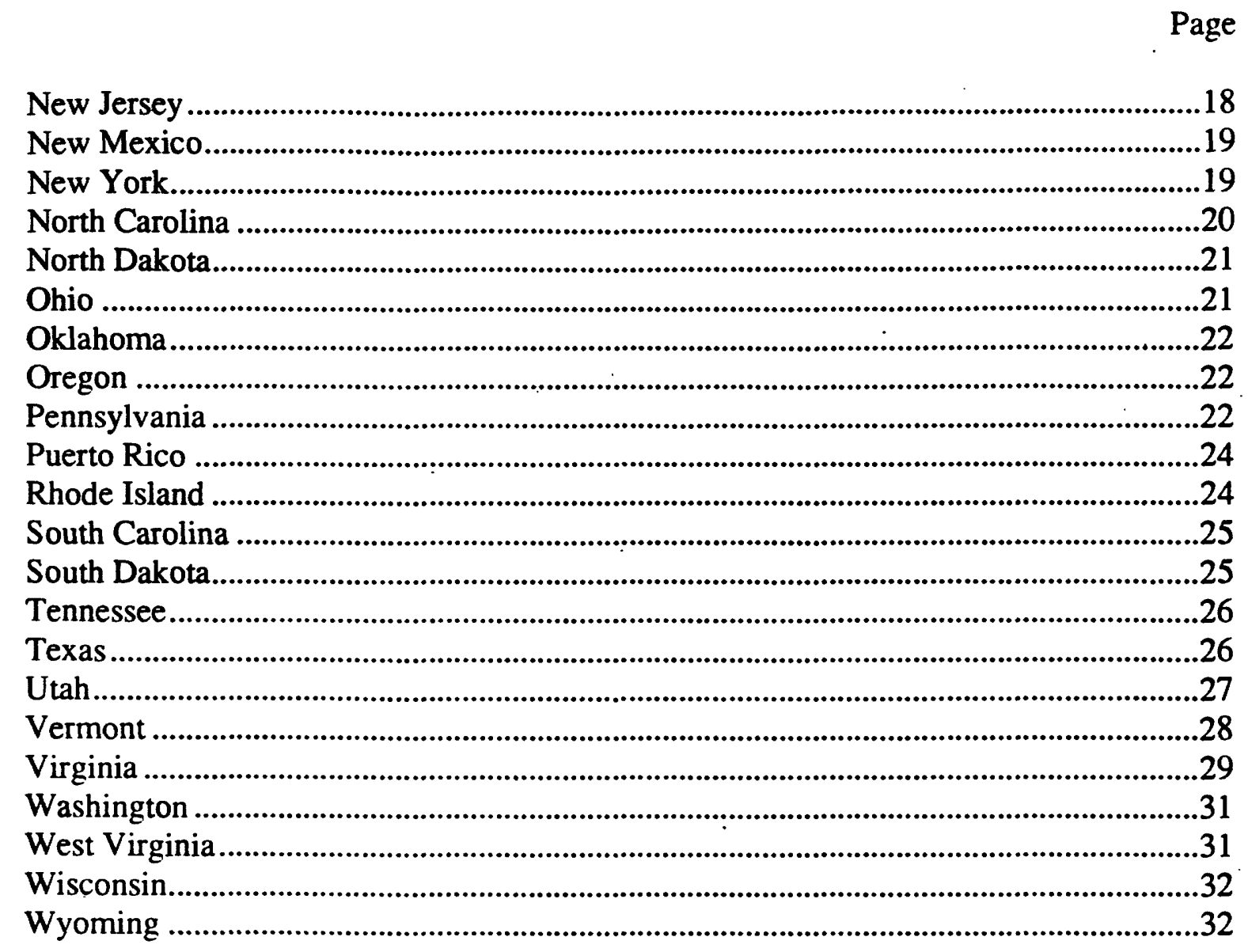

List of acronyms and abbreviations 


\title{
DIRECTORY OF ASSISTANCE CENTERS OF THE NATIONAL WATER DATA EXCHANGE (NAWDEX)
}

\author{
By Cassandra D. Blackwell
}

\begin{abstract}
The National Water Data Exchange (NAWDEX), managed by the U.S. Geological Survey, has established a network of Assistance Centers throughout the United States and Puerto Rico to assist users of water data in identifying and locating the data they need. This Directory provides the information needed to contact any of the established Centers.
\end{abstract}

\section{INTRODUCTION}

The National Water Data Exchange (NAWDEX) is a nationwide program managed by the U.S. Geological Survey to assist users of water data or water-related data in identifying, locating, and acquiring needed data. NAWDEX is a confederation of water-oriented organizations working together to make their data more readily accessible and to facilitate a more efficient exchange of water data. NAWDEX services are available through a Program Office located at the U.S. Geological Survey's National Center in Reston, Virginia, and a nationwide network of Assistance Centers established in 45 states, the District of Columbia and Puerto Rico to provide local and convenient access to NAWDEX facilities. This Directory provides the names of organizations and persons to contact, addresses, telephone numbers, and the various types of data and services available at each location.

\section{SERVICES AVAILABLE}

A variety of user services are provided by NAWDEX. These include assistance in identifying and locating needed water data and referring the requester to the organization that retains the data required. A Master Water Data Index is maintained which allows NAWDEX to locate those sites for which desired water data are available, and to provide the identifying information for each site necessary to obtain the data from the organization retaining it. A Water Data Sources Directory is also maintained that identifies organizations that are sources of water data and locations within these organizations from which data may be obtained.

NAWDEX services are available to any organization or individual.

\section{SERVICE CHARGES}

Charges for NAWDEX services are assessed at the option of the organization providing the requested data or data service. Search assistance services are provided free by NAWDEX to the greatest extent possible. Charges are assessed, however, for those requests requiring computer services, extensive personnel time, duplicating services, or service costs accrued by NAWDEX 
from other sources in the course of providing services. In all cases, charges assessed by NAWDEX Assistance Centers will not exceed the direct costs incurred in responding to the data request. Estimates of cost are provided by NAWDEX upon request and in all cases where costs are anticipated to be substantial.

\section{ADDITIONAL INFORMATION}

The participation of private organizations as NAWDEX Assistance Centers does not imply endorsement by the U.S. Geological Survey of any organization, service, system, or product within the private sector. Rather, the NAWDEX program serves as a public forum for informing its users of data services and products available from all sectors of the water-data community. For additional information concerning the NAWDEX program or its services, contact:

Program Office

National Water Data Exchange (NAWDEX)

U.S. Geological Survey

421 National Center

12201 Sunrise Valley Drive

Reston, Virginia 22092

Telephone: (703) 648-6848

cdblack@nxdxqvarsa.er.usgs.gov 


\section{LOCATIONS OF NAWDEX ASSISTANCE CENTERS}

\section{ALABAMA}

ORGANIZATION: U.S. Geological Survey, Water Resources Division ADDRESS: 520 19th Avenue, Tuscaloosa, AL 35401

TELEPHONE: (205) 752-8104 Central Time

FAX: (205) 752-9086

NAWDEX CONTACT: Will Mooty

AVAILABLE DATA FROM ORGANIZATION:

Types of Data: Surface Water, Water Quality, Ground Water, Precipitation

Geographical Coverage: State of Alabama

Media in Which Data Stored: Machine readable, Hard copy files, Published reports

Types of Media Output: Computer printout, Published (Printed) reports

MAJOR COMPUTERIZED DATA SYSTEM(S) ACCESSED: NAWDEX, WATSTORE DESCRIPTION OF EQUIPMENT USED: PRIME Model 9955

\section{ALASKA}

ORGANIZATION: U.S. Geological Survey, Water Resources Division ADDRESS: 4230 University Drive, Suite 201, Anchorage, AK 99508-4664

TELEPHONE: (907) 786-7100

Alaska Time (Pacific time minus 1 hour)

FAX: (907) 786-7150

NAWDEX CONTACT: Liska Snyder

AVAILABLE DATA FROM ORGANIZATION:

Types of Data: Surface Water, Water Quality, Ground Water

Geographical Coverage: State of Alaska

Media in Which Data Stored: Published reports, Computer files, Hard copy files

Types of Media Output: Computer printout, Published (Printed) reports, Floppy disks MAJOR COMPUTERIZED DATA SYSTEM(S) ACCESSED: NAWDEX, WATSTORE DESCRIPTION OF EQUIPMENT USED: PRIME 9955 Model II, Data General 6220 server ADDITIONAL COMPUTER SERVICES OR OTHER INFORMATION PRODUCTS PROVIDED BY ORGANIZATION: Project Reports (as long as surplus printed copy available)

\section{ARIZONA}

ORGANIZATION: U.S. Geological Survey, Water Resources Division ADDRESS: 375 S. Euclid Avenue, Tucson, AZ 85719 TELEPHONE: (602) 670-6120 Mountain Time

FAX: (602) 67()-5592

NAWDEX CONTACT: Christopher Smith 
AVAILABLE DATA FROM ORGANIZATION:

Types of Data: Surface Water, Water Quality, Ground Water

Geographical Coverage: State of Arizona

Media in Which Data Stored: Machine readable, Hard copy files, Published reports

Types of Media Output: Magnetic tape, Magnetic disk, Computer printout, Published (Printout) reports, Graphics

MAJOR COMPUTERIZED DATA SYSTEM(S) ACCESSED: NAWDEX, WATSTORE, DESCRIPTION OF EQUIPMENT USED: PRIME 750, Calcomp Plotter, various interactive terminals

\section{ARKANSAS}

ORGANIZATION: U.S. Geological Survey, Water Resources Division

ADDRESS: 401 Hardin Road, Little Rock, AR 72211

TELEPHONE: (501) 228-3639 Central Time

FAX: (501) 228-3601

NAWDEX CONTACT: John E. Owen

AVAILABLE DATA FROM ORGANIZATION:

Types of Data: Surface Water, Water Quality, Ground Water, Precipitation

Geographical Coverage: State of Arkansas

Media in Which Data Stored: Machine readable, Hard copy files, Published reports

Types of Media Output: Computer printout, Published (Printed) reports

MAJOR COMPUTERIZED DATA SYSTEM(S) ACCESSED: NAWDEX, WATSTORE, STORET, ADAPS

DESCRIPTION OF EQUIPMENT USED: PRIME Mini-Computer System and associated equipment

\section{CALIFORNIA}

ORGANIZATION: U.S. Geological Survey, Water Resources Division

ADDRESS: Room 2234 Federal Building, 2800 Cottage Way, Sacramento, CA 95825

TELEPHONE: (916) 978-4643

Pacific Time

FAX: (916) 978-5529

NAWDEX CONTACT: John Bader, Volunteer

AVAILABLE DATA FROM ORGANIZATION:

Types of Data: Surface Water, Water Quality, Ground Water

Geographical Coverage: State of California

Media in Which Data Stored: Published reports, Computer files

Types of Media Output: Magnetic tape, Computer printout, Published (Printed) reports, Magnetic disk

MAJOR COMPUTERIZED DATA SYSTEM(S) ACCESSED: NAWDEX, WATSTORE DESCRIPTION OF EQUIPMENT USED: PRIME Computer, TAB terminal 


\section{CALIFORNIA--Continued}

ORGANIZATION: Earth Science Information Center, U.S. Geological Survey

ADDRESS: Room 3128, Building 3 (MS 533), 345 Middlefield Road, Menlo Park, CA 94025

TELEPHONE: (415) 329-4390

Pacific Time

FAX: (415) 329-5130

NAWDEX CONTACT: Jack Mottram

AVAILABLE DATA FROM ORGANIZATION:

Types of Data: Surface Water, Water Quality, Ground Water

Geographical Coverage: States of Alaska, Arizona, California, Hawaii, Idaho, Nevada,

Oregon, Utah, Washington

Media in Which Data Stored: Hard copy files, Published reports

Types of Media Output: Ad hoc bibliographics

MAJOR COMPUTERIZED DATA SYSTEM(S) ACCESSED: PUBMANUS

DESCRIPTION OF EQUIPMENT USED: Video terminal and printer

\section{COLORADO}

ORGANIZATION: U.S. Geological Survey, Water Resources Division

ADDRESS: Building 53, Denver Federal Center, Mail Stop 415, Box 25046, Lakewood, CO 80225

TELEPHONE: (303) 236-4882

Mountain Time

FAX: (303) 236-4912

NAWDEX CONTACT: Vacant

AVAILABLE DATA FROM ORGANIZATION:

Types of Data: Surface Water, Water Quality, Ground Water

Geographical Coverage: State of Colorado

Media in Which Data Stored: Machine readable, Hard copy files, Microform,

Published reports

Types of Media Output: Magnetic tape, Computer printout, Published (Printed) reports, Floppy disk

MAJOR COMPUTERIZED DATA SYSTEM(S) ACCESSED: WATSTORE, NWIS DESCRIPTION OF EQUIPMENT USED: PRIME Model 9955

ADDITIONAL COMPUTER SERVICES OR OTHER INFORMATION PRODUCTS

PROVIDED BY ORGANIZATION: Daily Values Hydrographs

\section{CONNECTICUT}

ORGANIZATION: U.S. Geological Survey, Water Resources Division

ADDRESS: Abraham A. Ribicoff Federal Building, 450 Main Street, Room 525, Hartford, CT 061(1)3

TELEPHONE: (203) 24()-3060)

Eastern Time

FAX: (203) 24()-3783

NAWDEX CONTACT: Jacob Bohr 
AVAILABLE DATA FROM ORGANIZATION: Water Resources, Hydrologic Data Types of Data: Surface Water, Water Quality, Ground Water, Precipitation Geographical Coverage: State of Connecticut

Media in Which Data Stored: Machine readable, Hard copy files, Published reports

Types of Media Output: Computer printout, Published (Printed) reports, Graphics MAJOR COMPUTERIZED DATA SYSTEM(S) ACCESSED: NAWDEX, WATSTORE, STORET

DESCRIPTION OF EQUIPMENT USED: Six WYSE, 4 TAB and 2 Graphon terminals. One Tektronix Graphics terminal. Four PC's, 4 Laser printers, numerous line printers. One Zeta plotter, 1 Hewlett Packard Plotter, and 1 Hitachi CD-ROM Drive. Four Data General workstations, 1 Server, and 1 Silcon Graphics U5, 7 x-Terminals, 2 Laser Printers.

\section{DELAWARE}

(See U.S. Geological Survey Office in Maryland)

\section{DISTRICT OF COLUMBIA}

ORGANIZATION: U.S. Geological Survey, Water Resources Division ADDRESS: 1849 C Street. NW, Room 2650, Washington, DC 20240 TELEPHONE: (202) 208-4(1)47 Eastern Time

FAX: (202) 208-6297

NAWDEX CONTACT: Stephen P. Shivers

AVAILABLE DATA FROM ORGANIZATION:

Types of Data: Surface Water, Water Quality, Ground Water

Geographical Coverage: All States

Media in Which Data Stored: Hard copy files, Published reports

Types of Media Output: Ad hoc bibliographies

\section{FLORIDA}

ORGANIZATION: U.S. Geological Survey, Water Resources Division ADDRESS: 227 N. Bronough Street. Suite 3015, Tallahassee, FL 32301 TELEPHONE: (904) 942-950) Eastern Time

FAX: (904) 942-9521

NAWDEX CONTACT: Linda Geiger 
AVAILABLE DATA FROM ORGANIZATION:

Types of Data: Surface Water, Water Quality, Ground Water, Precipitation, Meteorological

Geographical Coverage: State of Florida

Media in Which Data Stored: Machine readable, Hard copy files, Published reports

Types of Media Output: Magnetic tape, Magnetic disk, Computer printout, Published (Printed) reports, Graphics

MAJOR COMPUTERIZED DATA SYSTEM(S) ACCESSED: NAWDEX, WATSTORE, STORET

DESCRIPTION OF EQUIPMENT USED: PRIME Model 9955

ORGANIZATION: U.S. Geological Survey, Water Resources Division

ADDRESS: 9100 NW 36th Street, Suite 106, Miami, FL 33178

TELEPHONE: (305) 594-0655

Eastern Time

FAX: (305) 526-2881

NAWDEX CONTACT: Russell E. Curtis

AVAILABLE DATA FROM ORGANIZATION:

Types of Data: Surface Water, Water Quality, Ground Water

Geographical Coverage: Southern Florida

Media in Which Data Stored: Machine readable, Hard copy files, Published reports

Types of Media Output: Computer printout, Published (Printed) reports, Graphics

MAJOR COMPUTERIZED DATA SYSTEM(S) ACCESSED: NAWDEX, WATSTORE, STORET, HISARS

DESCRIPTION OF EQUIPMENT USED: PRIME Model 9955 Model II, Data General workstations

ORGANIZATION: U.S. Geological Survey. Water Resources Division

ADDRESS: 224 West Central Parkway. Suite 1(006, Altamonte Springs, FL 32714

TELEPHONE: (407) 865-7575

Eastern Time

FAX: $(407) 865-6733$

NAWDEX CONTACT: Richard Craig

AVAILABLE DATA FROM ORGANIZATION:

Types of Data: Surface Water, Water Quality, Ground Water

Geographical Coverage: North and East Florida

Media in Which Data Stored: Machine readable, Hard copy files

Types of Media Output: Magnetic tape. Magnetic disk, Computer printout, Published (Printed) reports 


\section{FLORIDA--Continued}

MAJOR COMPUTERIZED DATA SYSTEM(S) ACCESSED: NAWDEX, WATSTORE, MIS

DESCRIPTION OF EQUIPMENT USED: PRIME Model 9955 Model II, IBM-PC/486, Data General 6220 server, Data General workstations

ORGANIZATION: U.S. Geological Survey, Water Resources Division

ADDRESS: 4710 Eisenhower Boulevard, Suite B-5, Tampa, FL 33634

TELEPHONE: (813) 228-2124

Eastern Time

FAX: (813) 228-2274

NAWDEX CONTACT: Janice Todd

AVAILABLE DATA FROM ORGANIZATION:

Types of Data: Surface Water, Water Quality, Ground Water

Geographical Coverage: West-Central Florida

Media in Which Data Stored: Machine readable, Hard copy files, Published reports

Types of Media Output: Computer printout, 3.5 and 5.25 inch diskette in ASCII format, Published (Printed) reports

MAJOR COMPUTERIZED DATA SYSTEM(S) ACCESSED: NAWDEX, WATSTORE, NWIS, (local) QWDATA, ADAPS

DESCRIPTION OF EQUIPMENT USED: PRIME Model 9955 Model II, PC/AT-IBM Compatible

\section{GEORGIA}

ORGANIZATION: U.S. Geological Survey, Water Resources Division

ADDRESS: 3030 Amwiler Road, Suite 130, Peachtree Business Center, Atlanta, GA 30360 TELEPHONE: (404) 903-9100)

Eastern Time

FAX: (404) 9013-9199

NAWDEX CONTACT: Keith W. McFadden

AVAILABLE DATA FROM ORGANIZATION:

Types of Data: Surface Water. Water Quality, Ground Water

Geographical Coverage: State of Georgia

Media in Which Data Stored: Machine readable, Hard copy files, Published reports

Types of Media Output: Magnetic tape. Computer printout, Published (Printed) reports, Graphics

MAJOR COMPUTERIZED DATA SYSTEM(S) ACCESSED: NAWDEX, WATSTORE, NWIS

DESCRIPTION OF EQUIPMENT USED: PRIME 9955 Model II 


\section{HAWAII}

ORGANIZATION: U.S. Geological Survey, Water Resources Division

ADDRESS: 677 Ala Moana Boulevard, Honolulu, HI 96813-5412

TELEPHONE: (808) 522-8292

Alaska-Hawaii Time

FAX: (808) 522-8298

NAWDEX CONTACT: Iwao Matsuoka

AVAILABLE DATA FROM ORGANIZATION:

Types of Data: Surface Water, Water Quality, Ground Water,Precipitation

Geographical Coverage: Hawaiian Islands, Trust Territory of the Pacific Islands,

Guam, American Samoa, and Northern Mariana Islands

Media in Which Data Stored: Machine readable, Published reports

Types of Media Output: Computer printout, Published (Printed) reports

MAJOR COMPUTERIZED DATA SYSTEM(S) ACCESSED: NAWDEX, WATSTORE, STORET

DESCRIPTION OF EQUIPMENT USED: PRIME Computer

\section{IDAHO}

ORGANIZATION: U.S. Geological Survey, Water Resources Division

ADDRESS: 230 Collins Road, Boise, ID 83702

TELEPHONE: (208) 387-1300

Mountain Time

FAX: (208) 387-1372

NAWDEX CONTACT: Luther C. Kjelstrom

AVAILABLE DATA FROM ORGANIZATION:

Types of Data: Surface Water, Water Quality, Ground Water

Geographical Coverage: State of Idaho

Media in Which Data Stored: Machine readable, Hard copy files, Published reports

Types of Media Output: Computer printout, Published (Printed) reports

MAJOR COMPUTERIZED DATA SYSTEM(S) ACCESSED: NAWDEX, WATSTORE, STORET

DESCRIPTION OF EQUIPMENT USED: PRIME computer, Tektronix graphics, Calcomp plotter

\section{ILLINOIS}

ORGANIZATION: U.S. Geological Survey, Water Resources Division ADDRESS: 2204 Griffith Drive, Champaign, IL 61820

TELEPHONE: (217) 333-4952

Central Time

FAX: (217) 333-6540)

NAWDEX CONTACT: Robert A. Sinclair 


\section{ILLINOIS--Continued}

AVAILABLE DATA FROM ORGANIZATION:

Types of Data: Surface Water, Water Quality, Ground Water, Precipitation, Meteorological

Geographical Coverage: State of Illinois

Media in Which Data Stored: Machine readable, Hard copy files, Microform

Published reports

Types of Media Output: Magnetic Tape, Magnetic Disk, Computer printout, Graphics (IGIS), Floppy Disk

MAJOR COMPUTERIZED DATA SYSTEM(S) ACCESSED: NAWDEX, WATSTORE, STORET, Illinois Geographic Information System (IGIS)

DESCRIPTION OF EQUIPMENT USED: IBM 3081, IBM Personal Computers, and SUN SPARC Systems on TCP/IP Ethernet Data Communications Network

ORGANIZATION: U.S. Geological Survey, Water Resources Division

ADDRESS: Busey Bank County Plaza, Fourth Floor, 102 East Main Street, Urbana IL 61801 TELEPHONE: (217) 344-3002 Central Time

FAX: (217) 344-0082

NAWDEX CONTACT: Gary O. Balding

AVAILABLE DATA FROM ORGANIZATION:

Types of Data: Surface Water, Water Quality, Ground Water, Water Use

Geographical Coverage: State of Illinois

Media in Which Data Stored: Machine readable, Hard copy files, Published reports

Types of Media Output: Magnetic tape, Magnetic disk, Computer printout, Published (Printed) reports

MAJOR COMPUTERIZED DATA SYSTEM(S) ACCESSED: NAWDEX, WATSTORE, NWIS I

DESCRIPTION OF EQUIPMENT USED: PRIME 9955 mini computer with 32 megabytes of central memory, 3.0 gigabytes of disk storage, tape drive, line printer, and various PC's, MAC's, DG's and terminals. System is linked to more than 50 others via, USGS, WRD "DIS" network

ADDITIONAL COMPUTER SER VICES OR OTHER INFORMATION PRODUCTS PROVIDED BY ORGANIZATION: Various statistical and application programs including GIS 


\section{INDIANA}

ORGANIZATION: U.S. Geological Survey, Water Resources Division ADDRESS: 5957 Lakeside Boulevard, Indianapolis, IN 46278-1996

TELEPHONE: (317) 290-3333

Eastern Time

FAX: (317) 290-3313

NAWDEX CONTACT: Don Arvin

AVAILABLE DATA FROM ORGANIZATION:

Types of Data: Surface Water, Water Quality, Ground Water

Geographical Coverage: State of Indiana

Media in Which Data Stored: Machine readable, Hard copy files, Published reports

Types of Media Output: Computer printout, Published (Printed) reports, 3.5 inch disk

MAJOR COMPUTERIZED DATA SYSTEM(S) ACCESSED: NAWDEX, WATSTORE

DESCRIPTION OF EQUIPMENT USED: PRIME 9955, Line printer, Magnetic

tape 1600/6250 bpi, Data General

IOWA

ORGANIZATION: U.S. Geological Survey, Water Resources Division

ADDRESS: Room 269, Federal Building, 400 South Clinton, Box 1230, Iowa City, IA 52240

TELEPHONE: (319) 337-4191

Central Time

FAX: (319) 358-0510

NAWDEX CONTACT: Debra Sneck-Fahrer

AVAILABLE DATA FROM ORGANIZATION:

Types of Data: Surface Water, Water Quality, Ground Water

Geographical Coverage: State of lowa

Media in Which Data Stored: Machine readable, Hard copy files, Published reports

Types of Media Output: Computer printout, Published (Printed) reports

MAJOR COMPUTERIZED DATA SYSTEM(S) ACCESSED: NAWDEX, WATSTORE

DESCRIPTION OF EQUIPMENT USED: Multi-level work station including a PRIME computer and micros. 600 and 300 LPM printer.

Communicates with Survey Prime (DIS) and

University of Iowa Prime.

\section{KANSAS}

ORGANIZATION: U.S. Geological Survey, Water Resources Division

ADDRESS: 4821 Quail Crest Place, Lawrence, KS 66049-3839

TELEPHONE: (913) 832-3501

Central Time

FAX: (913) 832-350)

NAWDEX CONTACT: Claude Geiger 


\section{KANSAS--Continued}

AVAILABLE DATA FROM ORGANIZATION:

Types of Data: Surface Water, Water Quality, Ground Water

Geographical Coverage: State of Kansas

Media in Which Data Stored: Machine readable, Hard copy files, Published reports

Types of Media Output: Machine readable, Hard copy files, Published (Printed) reports MAJOR COMPUTERIZED DATA SYSTEM(S) ACCESSED: NAWDEX, WATSTORE, STORET

DESCRIPTION OF EQUIPMENT USED: PRIME 9955 Minicomputer System

\section{KENTUCKY}

ORGANIZATION: U.S. Geological Survey, Water Resources Division ADDRESS: 2301 Bradley Avenue, Louisville, KY 40217

TELEPHONE: (502) 582-5241

Eastern Time

FAX: (502) 582-5241

NAWDEX CONTATT: Sandy J. Couts or Harry C. Rollins AVAILABLE DATA FROM ORGANIZATION:

Types of Data: Surface Water, Water Quality, Ground Water

Geographical Coverage: State of Kentucky

Media in Which Data Stored: Machine readable, Hard copy files, Published reports

Types of Media Output: Magnetic tape, Magnetic disk, Computer printout, Published (Printed) reports

MAJOR COMPUTERIZED DATA SYSTEM(S) ACCESSED: NAWDEX, WATSTORE DESCRIPTION OF EQUIPMENT USED: PRIME Minicomputer

\section{LOUISIANA}

ORGANIZATION: U.S. Geological Survey, Water Resources Division ADDRESS: 3535 S. Sherwood Forest Boulevard, Suite 120, Baton Rouge, LA 70816 TELEPHONE: (504) 389-(281 Central Time

FAX: (504) 389-(17)6

NAWDEX CONTACT: Wendy Lovelace

AVAILABLE DATA FROM ORGANIZATION:

Types of Data: Surface Water. Water Quality, Ground Water, *Precipitation, *Meteorological (*in office records of NOAA), Geological

Geographical Coverage: State of Louisiana

Media in Which Data Stored: Machine readable as retrieved from NWIS and WATSTORE, Hard copy files, Microform (some), Published (Printed) reports, Graphics

Types of Media Output: Magnetic tape, Magnetic disk, Computer printout 


\section{LOUISIANA--Continued}

\section{MAJOR COMPUTERIZED DATA SYSTEM(S) ACCESSED: NAWDEX, WATSTORE, NWIS}

DESCRIPTION OF EQUIPMENT USED: PRIME Model 9955

ADDITIONAL COMPUTER SERVICES OR OTHER INFORMATION PRODUCTS

PROVIDED BY ORGANIZATION: Incidental information requests

\section{MAINE}

ORGANIZATION: U.S. Geological Survey, Water Resources Division

ADDRESS: 26 Ganneston Drive, Augusta, ME 04330

TELEPHONE: (207) 622-8201

Eastern Time

NAWDEX CONTACT: Joseph P. Nielsen

AVAILABLE DATA FROM ORGANIZATION: Surface water, ground water, and water-quality data. Copies of USGS reports published in Maine

Geographical Coverage: State of Maine

Media in which data stored: Computer printout

Types of Media Output: Paper copy. Computer disk (3.5 or 5.25 inch)

MAJOR COMPUTERIZED DATA SYSTEM(S) ACCESSED: NWIS, NWIS II, WATSTORE DESCRIPTION OF EQUIPMENT USED: PRIME minicomputer, DG Unix workstations, IBM PC's

\section{MARYLAND}

ORGA.NIZATION: U.S. Geological Survey. Water Resources Division

ADDRESS: 2(18 Cartoll Building. 86(1) LuS Salle Road. Towson, MD 21286

TELEPHONE: *(410) 512-48115 or $4 \$ 34 \quad$ Eastern Time

FAX: $(4101) 512-4 \times 111$

NAWDEX CONTACT: Rolkert W' James. Jr. or Paul E. Exter AV'AILABLE DATA FRON ()RC (i. VIZATION

Typer of Data: Surface M ster. Muler Quslus. Ground Water, *Precipitation.

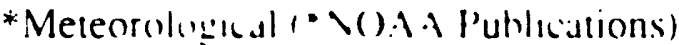

(ieographical Coverage: Mturyland. Deluuare. Dtstrict of Columbia

Nedia in Which Data Stored Hard cop! filen. Microform. Published reports

Types of Media Output: Matnettic tupe. Matnetic disk. Computer printout,

Mlicrofiche. Publushed (Printed) reports, Graphics, Punched cards

MAJOR COMPUTERIZED DATA SYSTEMIS S ACCESSED: NAWDEX, WATSTORE DESCRIPTION OF EQUIPMENT LSED. PRIME 9955 Model II 


\section{MASSACHUSETTS}

ORGANIZATION: U.S. Geological Survey, Water Resources Division

ADDRESS: 28 Lord Road, Suite 280, Marlborough, MA 01752

TELEPHONE: (508) 485-6360

Eastern Time

FAX: (508) 490-5068

NAWDEX CONTACT: Thomas B. Shepard

AVAILABLE DATA FROM ORGANIZATION:

Types of Data: Surface Water, Water Quality, Ground Water

Geographical Coverage: Massachusetts and Rhode Island

Media in Which Data Stored: Hard copy files, Microform, Published reports,

Magnetic tape, Floppy disk, Magnetic disk

Types of Media Output: Computer printout, Published (Printed) reports, Floppy disk MAJOR COMPUTERIZED DATA SYSTEM(S) ACCESSED: NAWDEX, NWIS, WATSTORE

DESCRIPTION OF EQUIPMENT USED: PRIME Model 6350, plus various PC's

\section{MICHIGAN}

ORGANIZATION: U.S. Geological Survey, Water Resources Division

ADDRESS: 6520 Mercantile Way, Suite 5, Lansing, MI 48911

TELEPHONE: (517) 887-8911 Eastern Time

FAX: (517) $377-1627$

NAWDEX CONTACT: Gary C. Huffman or Stephen P. Blumer AVAILABLE DATA FROM ORGANIZATION:

Types of Data: Surface Water. Water Quality, Ground Water

Geographical Coverage: Stute of Michigan

Media in Which Data Stored: Machine readable. Published reports

Types of Media Output: Computer printout. Published (Printed) reports MAJOR COMPUTERIZED DATA SYSTEM(S) ACCESSED: NAWDEX, WATSTORE DESCRIPTION OF EQUIPMENT USED: PC's. TAB terminals, Data General workstations ADDITIONAL COMPUTER SERVICES OR OTHER INFORMATION PRODUCTS

PROVIDED BY ORGANIZATION: Stutustical summaries of surface-water data

\section{MINNESOTA}

ORGANIZATION: U.S. Geological Survey. Water Resources Division ADDRESS: 2280 Woodale Drive. Mounds View. MN 55112

TELEPHONE: (612) 783-31(1)

Central Time

FAX: (612) 78.3-3111.3

NAWDEX CONTACT: Allan D. Arntson 


\title{
MINNESOTA--Continued
}

\begin{abstract}
AVAILABLE DATA FROM ORGANIZATION:
Types of Data: Surface Water, Water Quality, Ground Water

Geographical Coverage: State of Minnesota

Media in Which Data Stored: Hard copy files

Types of Media Output: Computer printout, Published (Printed) reports

MAJOR COMPUTERIZED DATA SYSTEM(S) ACCESSED: NAWDEX, WATSTORE, STORET
\end{abstract}

DESCRIPTION OF EQUIPMENT USED: PRIME Model 9955

\section{MISSISSIPPI}

ORGANIZATION: U.S. Geological Survey, Water Resources Division

ADDRESS: Suite 710, Federal Office Building, 100 West Capitol Street, Jackson, MS 39269 TELEPHONE: (601) 965-4600

Central Time

FAX: (601) 965-5782

NAWDEX CONTACT: Fred Morris, III

AVAILABLE DATA FROM ORGANIZATION:

Types of Data: Surface Water, Water Quality, Ground Water, Water Use

Geographical Coverage: State of Mississippi

Media in Which Data Stored: Machine readable, Hard copy files, Microform, Published reports, CD-ROM

Types of Media Output: Magnetic tape, Computer printout, Published (Printed) reports, Graphics, 3.5 and 5.25 inch diskettes

MAJOR COMPUTERIZED DATA SYSTEM(S) ACCESSED: NAWDEX, WATSTORE DESCRIPTION OF EQUIPMENT USED: PRIME, Houston DP-8 plotter, Hewlett Packard plotters, PC compatibles, Data General workstations, Macintosh

\section{MISSOURI}

ORGANIZATION: U.S. Geological Survey, Water Resources Division ADDRESS: 1400) Independence Road, Mail Stop 200, Rolla, MO 65401

TELEPHONE: (314) 341-0824

Central Time

FAX: (314) 341-0805

NAWDEX CONTACT: Loyd Waite

AVAILABLE DATA FROM ORGANIZATION:

Types of Data: Surface Water, Water Quality, Ground Water

Geographical Coverage: State of Missouri

Media in Which Data Stored: Machine readable, Hard copy files, Published reports

Types of Media Output: Magnetic tape, Computer printout, Published (Printed) reports MAJOR COMPUTERIZED DATA SYSTEM(S) ACCESSED: NAWDEX, WATSTORE, DESCRIPTION OF EQUIPMENT USED: PRIME Model 750 


\section{MONTANA}

ORGANIZATION: U.S. Geological Survey, Water Resources Division

ADDRESS: Federal Building, Drawer 10076, 301 So Park Avenue, Helena, MT 59626-0076

TELEPHONE: (406) 449-5263

Mountain Time

FAX: (406) 449-5497

NAWDEX CONTACT: Melvin White

AVAILABLE DATA FROM ORGANIZATION:

Types of Data: Surface Water, Water Quality, Ground Water, Precipitation

Geographical Coverage: State of Montana and some streamflow sites in the

Canadian Provinces of Alberta and Saskatchewan

Media in Which Data Stored: Hard copy files, Published reports

Types of Media Output: Magnetic disk, Computer printout, Published (Printed) reports, Magnetic tape

MAJOR COMPUTERIZED DATA SYSTEM(S) ACCESSED: NAWDEX, WATSTORE

DESCRIPTION OF EQUIPMENT USED: PRIME S2 System, Data General 6000

\section{NEBRASKA}

ORGANIZATION: Nebraska Natural Resources Commission

ADDRESS: 301 Centennial Mall South, P.O. Box 94876, Lincoln, NE 68509

TELEPHONE: (402) 471-2081 Central Time

FAX: (402) 471-3132

NAWDEX CONTACT: Mahendra K. Bansal, Head, Data Bank Section, Natural Resources Information System

AVAILABLE DATA FROM ORGANIZATION:

Types of Data: Surface Water, (streamflow, peakflow), Surface and Ground Water Quality, Ground Water levels, Drinking Water Sampling, Precipitation, Meteorological (climatological), Water Rights, Well Registration, Center Pivots, Agricultural Crops, Demographic, Limited Soil Surveys and Interpretations, Dams Inventory, Hydrological Delineations, Land Use, Canal Diversions, Reservoir Storage, Population Estimates, Decennial Census, and TIGER Line files, Wetlands Inventory.

Geographical Coverage: State of Nebraska

Media in Which Data Stored: Machine readable, Hard copy files, Published reports

Types of Media Output: Magnetic tape, Floppy disk, 8 millimeter tape, TK50 and optical disk cartridges. Computer printout, Published (Printed) reports, and computer generated maps

MAJOR COMPUTERIZED DATA SYSTEM(S) ACCESSED: WATSTORE, STORET, ESDD, NAWDEX, Nebraska Natural Resources Information System (NNRIS) 
DESCRIPTION OF EQUIPMENT USED: DEC 5000.240 File Server, DEC 5000/125's workstations, DEC server, LP37-VA line printer, CDROM, optical disk drive, Tangent and Howtek scanner and Kurta digitizer. CNX500 Proteon Bridge, IBM 3777/Model 1, various IBM compatible PC's and Calcomp 58000 and 5800 series color electrostatic plotters; Hewlett Packard 7586B Pen Plotter. The Data Bank is linked to the University of Nebraska, and the State-house Computer Systems and INTERNET through ETHERNET LAN and wide area networks ADDITIONAL COMPUTER SERVICES OR OTHER INFORMATION PRODUCTS PROVIDED BY ORGANIZATION: Direct data access facility through MOSAIC and GOPHER World-wide Web Servers is available to all users. GIS processing of data is provided using ARC/INFO and GRASS GIS software systems. Users are required to establish a computer account with the NRC Data Bank. Copies of the Users Manual, Information Guide, and Data Access Manual are available on request

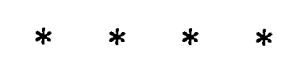

ORGANIZATION: U.S. Geological Survey, Water Resources Division ADDRESS: Room 406, Federal Building \& U.S. Courthouse, 100 Centennial Mall North, Lincoln, NE 68508

TELEPHONE: (402) 437-5082 Central Time

FAX: (402) 437-5139

NAWDEX CONTACT: Glen Engel

AVAILABLE DATA FROM ORGANIZATION:

Types of Data: Surface Water, Water Quality, Ground Water

Geographical Coverage: State of Nebraska

Media in Which Data Stored: Machine readable, Hard copy files, Published reports

Types of Media Output: Magnetic tape. Computer printout, Published (Printed) reports,

Graphics, (modern available for access if approved by

District Chief)

MAJOR COMPUTERIZED DATA SYSTEM(S) ACCESSED: NAWDEX, WATSTORE, Local System

DESCRIPTION OF EQUIPMENT USED: Calcomp 1044GT plotter, Mitron, 3 laser printers, Altek digitizer, 3 color graphics terminals and low resolution color graphics printers, COMPAQ 386/20) PC. Twenty Data General workstations, 2 QIC tape drives (150 mb), 2 postscript laser printers 


\section{NEVADA}

ORGANIZATION: U.S. Geological Survey, Water Resources Division

ADDRESS: Room 102, 333 West Nye Lane, Carson City, NV 89706 .

TELEPHONE: (702) 887-7600 Pacific Time

FAX: (702) 887-7629

NAWDEX CONTACT: $M$. Teresa Foglesong

AVAILABLE DATA FROM ORGANIZATION:

Types of Data: Surface Water, Water Quality, Ground Water, Water Use, various GIS

Themes

Geographical Coverage: State of Nevada

Media in Which Data Stored: Machine readable, Published reports

Types of Media Output: Magnetic disk (floppy), Magnetic tape, Computer printout,

Published (Printed) reports, Graphics

MAJOR COMPUTERIZED DATA SYSTEM(S) ACCESSED: NAWDEX, WATSTORE, NWIS

DESCRIPTION OF EQUIPMENT USED: PRIME Computer, Data General workstations

\section{NEW HAMPSHIRE}

(See U.S. Geological Survey Office in Massachusetts)

\section{NEW JERSEY}

ORGANIZATION: U.S. Geological Survey, Water Resources Division

ADDRESS: Mountain View Office Park, 810 Bear Tavern Road, Suite 206, West Trenton, NJ 08628

TELEPHONE: (609) 771-3900 Eastern Time

FAX: $(609) 771-3915$

NAWDEX CONTACT: Deloris W. Speight

AVAILABLE DATA FROM ORGANIZATION:

Types of Data: Surface Water, Water Quality, Ground Water,

Geographical Coverage: State of New Jersey

Media in Which Data Stored: Machine readable, Hard copy files, Microform, Published reports, Computer disk files

Types of Media Output: Magnetic tape, Magnetic disk, Computer printout, Microfiche, Published (Printed) reports, Graphics

MAJOR COMPUTERIZED DATA SYSTEM(S) ACCESSED: NAWDEX, NWIS, GIS

DESCRIPTION OF EQUIPMENT USED: PRIME 9955, Data General, PC's 


\section{NEW MEXICO}

ORGANIZATION: U.S. Geological Survey, Water Resources Division

ADDRESS: 4501 Indian School Road, NE, Suite 200, Albuquerque, NM 87110-3929

TELEPHONE: (505) 262-5300

Mountain Time

FAX: (505) 262-5398

NAWDEX CONTACT: Linda V. Beal

AVAILABLE DATA FROM ORGANIZATION:

Types of Data: Surface Water, Water Quality, Ground Water, Precipitation, Meteorological

Geographical Coverage: New Mexico and surrounding States

Media in Which Data Stored: Machine readable, Hard copy files, Published reports

Types of Media Output: Magnetic tape, Computer printout, Published (Printed) reports,

Graphics

MAJOR COMPUTERIZED DATA SYSTEM(S) ACCESSED: NAWDEX, WATSTORE

DESCRIPTION OF EQUIPMENT USED: PRIME Minicomputer with tape drive no card punch

\section{NEW YORK}

ORGANIZATION: New York State Geological Survey

ADDRESS: Room 3136 CEC, Albany, NY 12230

TELEPHONE: (518) 474-5816 Eastern Time

FAX: (518) 473-8496

NAWDEX CONTACT: Robert H. Fickies

AVAILABLE DATA FROM ORGANIZATION:

Types of Data: Bedrock geology, surficial geology, air photo and satellite imagery, petroleum well records, surface water, ground water

Geographical Coverage: New York State

Media in Which Data Stored: Published and unpublished maps and reports, Machine readable. Hard copy files

Types of Media Output: Published and unpublished maps and reports, Floppy disk, Computer printout

MAJOR COMPUTERIZED DATA SYSTEM(S) ACCESSED: NYGEODEX, NAWDEX, WATSTORE, ESDD

DESCRIPTION OF EQUIPMENT USED: IBM 386

ADDITIONAL COMPUTER SERVICES OR OTHER INFORMATION PRODUCTS

PROVIDED BY ORGANIZATION: Bedrock core library, ditch sample library, Publications list available upon request

ORGANIZATION: U.S. Geological Survey. Water Resources Division

ADDRESS: 445 Broadway, Room 343, P.O. Box 1669, Albany, NY 12201

TELEPHONE: (518) 472-3109

Eastern Time

FAX: (518) 472-2805

NAWDEX CONTACT: Lloyd A. Wagner 
AVAILABLE DATA FROM ORGANIZATION:

Types of Data: Surface Water, Water Quality, Ground Water

Geographical Coverage: State of New York

Media in Which Data Stored: Machine readable, Hard copy files, Published reports

Types of Media Output: Magnetic tape

MAJOR COMPUTERIZED DATA SYSTEM(S) ACCESSED: NAWDEX, WATSTORE

DESCRIPTION OF EQUIPMENT USED: PRIME Model 6350

ORGANIZATION: U.S. Geological Survey, Water Resources Division

ADDRESS: 2045 Route 112, Building 4, Coram, NY 11727

TELEPHONE: (516) 736-0783

Eastern Time

FAX: (516) 736-4283

NAWDEX CONTACT: George W. Hawkins

AVAILABLE DATA FROM ORGANIZATION:

Types of Data: Surface Water, Water Quality, Ground Water, Precipitation

Geographical Coverage: Long Island

Media in Which Data Stored: Machine readable, Hard copy files, Published reports

Types of Media Output: Magnetic tape, Magnetic disk, Computer printout,

Published (Printed) reports, Graphics

MAJOR COMPUTERIZED DATA SYSTEM(S) ACCESSED: NAWDEX, WATSTORE, NWIS

DESCRIPTION OF EQUIPMENT USED: PRIME 9955 Model II

\section{NORTH CAROLINA}

ORGANIZATION: U.S. Geological Survey, Water Resources Division

ADDRESS: 3916 Sunset Ridge Road. Raleigh, NC 27607

TELEPHONE: (919) 571-4014 Eastern Time

FAX: (919) 571-4(141

NAWDEX CONTACT: Pamilee L. Breton

AVAILABLE DATA FROM ORGANIZATION:

Types of Data: Surface Water, Water Quality, Ground Water, Precipitation (limited)

Geographical Coverage: State of North Carolina

Media in Which Data Stored: Machine readable, Hard copy files, Published reports

Types of Media Output: Magnetic tape. Magnetic disk, Computer printout, Published

(Printed) reports 
MAJOR COMPUTERIZED DATA SYSTEM(S) ACCESSED: USGS NC District Computer file, NAWDEX, WATSTORE

DESCRIPTION OF EQUIPMENT USED: Batch and Interactive Terminals, PRIME Model 9955, Data General workstations and dual processing server on Wide Area Network and Internet

ADDITIONAL COMPUTER SERVICES OR OTHER INFORMATION PRODUCTS PROVIDED BY ORGANIZATION: Flood-Prone Maps

\section{NORTH DAKOTA}

ORGANIZATION: U.S. Geological Survey, Water Resources Division

ADDRESS: 821 East Interstate Avenue, Bismarck, ND 58501

TELEPHONE: (701) 250-4604

Central Time

FAX: (701) 250-4252

NAWDEX CONTACT: Russell E. Harkness

AVAILABLE DATA FROM ORGANIZATION:

Types of Data: Surface Water, Water Quality, Ground Water

Geographical Coverage: State of North Dakota

Media in Which Data Stored: Machine readable, Hard copy files, Published reports

Types of Media Output: Floppy disk (3.5 and 5.25 inch), Computer printout, Published (Printed) reports, Graphics

MAJOR COMPUTERIZED DATA SYSTEM(S) ACCESSED: NAWDEX, WATSTORE

DESCRIPTION OF EQUIPMENT USED: PRIME Model 9955 and Data General Aviion 300

\section{$\mathrm{OHIO}$}

ORGANIZATION: U.S. Geological Survey, Water Resources Division

ADDRESS: 975 West Third Avenue, Columbus, $\mathrm{OH} 43212$

TELEPHONE: (614) 469-5553

Eastern Time

FAX: (614) $469-5626$

NAWDEX CONTACT: Ron Veley

AVAILABLE DATA FROM ORGANIZATION:

Types of Data: Surface Water, Water Quality, Ground Water, Precipitation (limited), GWSI, Peak, Water Level. Basin Characteristics, Water Use

Geographical Coverage: State of Ohio

Media in Which Data Stored: Machine readable, Hard copy files, Published reports

Types of Media Output: Computer printout, Published (Printed) reports, Magnetic media MAJOR COMPUTERIZED DATA SYSTEM(S) ACCESSED: NAWDEX, WATSTORE DESCRIPTION OF EQUIPMENT USED: PRIME Model 9955, IBM PC compatible, AppleMacintosh, Data General Aviion (UNIX)

ADDITIONAL COMPUTER SERVICES OR OTHER INFORMATION PRODUCTS PROVIDED BY ORGANIZATION: Ohio Flood-Prone Area Maps 


\section{OKLAHOMA}

ORGANIZATION: U.S. Geological Survey, Water Resources Division

ADDRESS: Broadway Executive Park, Building 7, 202 NW 66th, Oklahoma City, OK 73116 TELEPHONE: (405) 231-4256

Central Time

FAX: (405) 231-5079

NAWDEX CONTACT: John S. Havens

AVAILABLE DATA FROM ORGANIZATION:

Types of Data: Surface Water, Water Quality, Ground Water, Precipitation (Miscellaneous)

Geographical Coverage: State of Oklahoma

Media in Which Data Stored: Hard copy files, Published reports, Disk, Tape

Types of Media Output: Computer printout, Published (Printed) reports, Magnetic tape, Floppy disk

MAJOR COMPUTERIZED DATA SYSTEM(S) ACCESSED: NAWDEX, NWIS, STORET, WATSTORE

DESCRIPTION OF EQUIPMENT USED: PRIME Model 9955

\section{OREGON}

ORGANIZATION: U.S. Geological Survey, Water Resources Division

ADDRESS: 10615 S.E. Cherry Blossom Drive, Portland, OR 97216

TELEPHONE: (503) 251-3201

Pacific Time

FAX: (503) 251-3470

NAWDEX CONTACT: Suzanne J. Miller

AVAILABLE DATA FROM ORGANIZATION:

Types of Data: Surface Water, Water Quality, Ground Water

Geographical Coverage: State of Oregon

Media in Which Data Stored: Machine readable, Hard copy files, Microform, Published reports

Types of Media Output: Computer printout, Published (Printed) reports MAJOR COMPUTERIZED DATA SYSTEM(S) ACCESSED: NAWDEX, WATSTORE DESCRIPTION OF EQUIPMENT USED: PRIME Model 9955

\section{PENNSYLVANIA}

ORGANIZATION: U.S. Geological Survey, Water Resources Division ADDRESS: 840 Market Street, Lemoyne, PA 17043

TELEPHONE: (717) 730-6916

Eastern Time

FAX: (717) 730-6997

NAWDEX CONTACT: Robert Helm 


\section{PENNSYLVANIA--Continued}

AVAILABLE DATA FROM ORGANIZATION:

Types of Data: Surface Water, Flow Statistics and Water Quality, Ground Water location, Quantity and Quality

Geographical Coverage: State of Pennsylvania

Media in Which Data Stored: Machine readable, Hard copy files, Published reports

Types of Media Output: Magnetic tape, Floppy disk, Computer printout, Published (Printed) reports

MAJOR COMPUTERIZED DATA SYSTEM(S) ACCESSED: ADAPS, QWDATA, GWSI, WATSTORE

DESCRIPTION OF EQUIPMENT USED: PRIME Model 9955, IBM-compatible microcomputer, Data General workstations

ORGANIZATION: U.S. Geological Survey, Water Resources Division

ADDRESS: Great Valley Corporate Center, 111 Great Valley Parkway, Malvern, PA 19355 TELEPHONE: (215) 647-9008 Eastern Time

FAX: (215) 647-4594

NAWDEX CONTACT: Curtis L. Schreffler

AVAILABLE DATA FROM ORGANIZATION:

Types of Data: Surface Water, Water Quality, Ground Water, Precipitation

Geographical Coverage: Delaware River Basin

Media in Which Data Stored: Machine readable, Hard copy files, Published reports

Types of Media Output: Computer printout, Published (Printed) reports, Diskette MAJOR COMPUTERIZED DATA SYSTEM(S) ACCESSED: NAWDEX, WATSTORE, NWIS, GWSI

DESCRIPTION OF EQUIPMENT USED: Dell 200 PC AT, IBM XT Graphon 140, l Alter digitizer with HP Plotter, Envirolab tape reader, 6 Zenith Lap Top PC's, 24 Hlandar Telemetry Platforms, 62 Synergetic Telemetry Platforms, 19 Pressure Sensors, PRIME terminals, Data General workstations, 2 Data General x-Terminals, 1 Gateway 2000 Pentium PC

ORGANIZATION: U.S. Geological Survey, Water Resources Division

ADDRESS: Room 2204 Moorhead Federal Building, 1000 Liberty Avenue, Pittsburgh, PA 15222

TELEPHONE: (412) 644-2864 Eastern Time FAX: (412) 355-2548

NAWDEX CONTACT: Greg Wehner 


\section{PENNSYLVANIA--Continued}

\section{AVAILABLE DATA FROM ORGANIZATION:}

Types of Data: Surface Water, Water Quality, Ground Water Geographical Coverage: Western Pennsylvania

Media in Which Data Stored: Machine readable, Hard copy files, Published reports

Types of Media Output: Computer printout, Published (Printed) reports

MAJOR COMPUTERIZED DATA SYSTEM(S) ACCESSED: NAWDEX, WATSTORE

DESCRIPTION OF EQUIPMENT USED: TAB 132/15 terminal, Centronics printer, Hewlett

Packard 7475A plotter, Hewlett Packard 7570A

Draftpro Plotter, Sun 4/110 microcomputers, IBMcompatible microcomputers, Hewlett Packard Laser printers, Postscript printers, Data General workstations

\section{PUERTO RICO (includes Virgin Islands)}

ORGANIZATION: U.S. Geological Survey, Water Resources Division

ADDRESS: P.O. Box 364424, San Juan, PR 00936-4424

TELEPHONE: (809) 749-4346

Atlantic Time

FAX: (809) 749-4462

NAWDEX CONTACT: Teresa Dopazo or Vicki Sanchez

AVAILABLE DATA FROM ORGANIZATION:

Types of Data: Surface Water, Water Quality, Ground Water, Meteorological

Geographical Coverage: Puerto Rico and the U.S. Virgin Island

Media in Which Data Stored: Machine readable, Hard copy files, Published reports

Types of Media Output: Magnetic tape, Computer printout, Published (Printed) reports, MAJOR COMPUTERIZED DATA SYSTEM(S) ACCESSED: NAWDEX, WATSTORE, Inhouse

DESCRIPTION OF EQUIPMENT USED: PRIME 9955 Model II, IBM Personal computers, Data General workstations

\section{RHODE ISLAND}

(See U.S. Geological Survey Office in Massachusetts) 


\section{SOUTH CAROLINA}

ORGANIZATION: South Carolina Water Resources Commission

ADDRESS: 1201 Main Street, Suite 1100 Capital Center, Columbia, SC 29201

TELEPHONE: (803) 737-0800

Eastern Time

FAX: (803) 765-9080

NAWDEX CONTACT: Rick Wrigley, Computer Systems Manager

AVAILABLE DATA FROM ORGANIZATION:

Types of Data: Water Quality, Ground Water, Water Use Data, Weather Data

Geographical Coverage: State of South Carolina

Media in Which Data Stored: Hard copy files, Published, Computer files

Types of Media Output: Published (Printed), Computer generated reports

MAJOR COMPUTERIZED DATA SYSTEM(S) ACCESSED: SAS, DBASE, ARC/INFO:

DESCRIPTION OF EQUIPMENT USED: IBM PC's/AT, modem-dial-up capability, VAX 8350, Micro VAX, VAX 3500, VAX 4000

ORGANIZATION: U.S. Geological Survey, Water Resources Division

ADDRESS: Stephenson Center, Suite 129, 720 Gracern Road, Columbia SC 29210-7651

TELEPHONE: (803) 750-6100

Eastern Time

FAX: (803) 750-6181

NAWDEX CONTACT: David E. Bower

AVAILABLE DATA FROM ORGANIZATION:

Types of Data: Surface Water, Water Quality, Ground Water

Geographical Coverage: State of South Carolina

Media in Which Data Stored: Machine readable, Hard copy files, Published reports

Types of Media Output: Computer printout, Published (Printed) reports

MAJOR COMPUTERIZED DATA SYSTEM(S) ACCESSED: WATSTORE, NAWDEX

DESCRIPTION OF EQUIPMENT USED: PRIME Computer

\section{SOUTH DAKOTA}

ORGANIZATION: U.S. Geological Survey, Water Resources Division

ADDRESS: $16(18$ Mountain View. Rapid City, SD 57702

TELEPHONE: (605) 394-178(), ext. $222 \quad$ Mountain Time

FAX: (605) 394-5373

NAWDEX CONTACT: Ralph W. Teller

AVAILABLE DATA FROM ORGANIZATION:

Types of Data: Surface Water. Water Quality, Ground Water

Geographical Coverage: State of South Dakota

Media in Which Data Stored: Machine readable, Hard copy files, Microform,

Published reports

Types of Media Output: Computer printout, Published (Printed) reports, Graphics 
SOUTH DAKOTA--Continued

MAJOR COMPUTERIZED DATA SYSTEM(S) ACCESSED: NAWDEX, WATSTORE,
STORET

DESCRIPTION OF EQUIPMENT USED: PRIME Model 9955 II and Data General Aviion 300,530

\section{TENNESSEE}

ORGANIZATION: U.S. Geological Survey, Water Resources Division

ADDRESS: 810 Broadway, Suite 500, Nashville, TN 37203

TELEPHONE: (615) 736-5424 ext. $3166 \quad$ Central Time

FAX: (615) 736-2066

NAWDEX CONTACT: Donna F. Flohr

AVAILABLE DATA FROM ORGANIZATION:

Types of Data: Surface Water, Water Quality, Ground Water, Precipitation

Geographical Coverage: State of Tennessee

Media in Which Data Stored: Machine readable, Hard copy files, Microform,

Published reports

Types of Media Output: Computer printout, Published (Printed) reports, Tape, Floppy disk MAJOR COMPUTERIZED DATA SYSTEM(S) ACCESSED: NAWDEX, WATSTORE, STORET

DESCRIPTION OF EQUIPMENT USED: PRIME 9955 Model II, Hewlett-Packard 758B plotter, Numonics Digitizer, IBM compatible PC's

\section{TEXAS}

ORGANIZATION: Texas Natural Resources Information System

ADDRESS: P.O. Box 13231, Austin, TX 78711-3231

TELEPHONE: (512) 463-84(12

Central Time

FAX: (512) 463-9893

NAWDEX CONTACT: Dr. Charles Palmer

AVAILABLE DATA FROM ORGANIZATION:

Types of Data: Surface Water. Water Quality, Ground Water, Precipitation, Meteorological

Geographical Coverage: State of Texas

Media in Which Data Stored: Machine readable, Hard copy files, Microform,

Published reports

Types of Media Output: Magnetic tape. Computer printout, Microfiche,

Published (Printed) reports, Graphics

MAJOR COMPUTERIZED DATA SYSTEM(S) ACCESSED: NWIS-II

DESCRIPTION OF EQUIPMENT USED: Univac 1100/62 computer, Disk, 6250 BPI tapes 


\section{UTAH}

ORGANIZATION: Center for Water Resources Research

ADDRESS: Utah State University, UMC-82, Logan, UT 84322

TELEPHONE: (801) $797-3155$ or 3172

Mountain Time

FAX: (801) 797-3663

NAWDEX CONTACT: David G. Tarboton

AVAILABLE DATA FROM ORGANIZATION:

Types of Data: Surface Water, Water Quality, Ground Water, Precipitation, Meteorological

Geographical Coverage: State of Utah (Northern)

Media in Which Data Stored: Machine readable, Hard copy files, Published reports

Types of Media Output: Magnetic disk, Computer printout, Published (Printed) reports MAJOR COMPUTERIZED DATA SYSTEM(S) ACCESSED: STORET

DESCRIPTION OF EQUIPMENT USED: Data General Unix workstations, Macintosh, PC

ORGANIZATION: Utah Division of Water Rights

ADDRESS: Room 223, 1636 West North Temple, Salt Lake City, UT 84116

TELEPHONE: (801) 538-7392

Mountain Time

FAX: (501) 538-7315

NAWDEX CONTACT: William Smart

AVAILABLE DATA FROM ORGANIZATION:

Types of Data: Surface Water, Ground Water, Water Rights, Well Logs

Geographical Coverage: State of Utah

Media in Which Data Stored: Hard copy files, Microform, Published reports

Types of Media Output: Magnetic tape, Magnetic disk, Computer printout, Graphics

MAJOR COMPUTERIZED DATA SYSTEM(S) ACCESSED: NAWDEX, WATSTORE

DESCRIPTION OF EQUIPMENT USED: Hewlett-Packard 3000 Series $947 \mathrm{LX}$ computer

ORGANIZATION: U.S. Bureau of Land Management, Fish and Wildlife Department

ADDRESS: Aquatic Ecosystem Monitoring Center, Fish and Wildlife Department, Utah State University, Logan. UT 84322-5210

TELEPHONE: (801) 750-2038 Mountain Time

FAX: (501) 750)-3798

NAWDEX CONTACT: Mark Vinson

AVAILABLE DATA FROM ORGANIZATION:

Types of Data: Surface Water, Water Quality, Precipitation, Aquatic Invertebrate data

Geographical Coverage: Western United States

Media in Which Data Stored: Published reports, Computer-accessed printouts

Types of Media Output: Magnetic disk, Computer printout, Published reports 
UTAH--Continued

\section{MAJOR COMPUTERIZED DATA SYSTEM(S) ACCESSED: NAWDEX, STORET, WATSTORE}

DESCRIPTION OF EQUIPMENT USED: Personal computers

ORGANIZATION: U.S. Geological Survey, Water Resources Division

ADDRESS: Room 1016, Administration Building, 1745 West 1700 South, Salt Lake City, UT 84104

TELEPHONE: (801) 975-3350

Mountain Time

FAX: (501) 975-3424

NAWDEX CONTACT: Julane Mulder

AVAILABLE DATA FROM ORGANIZATION:

Types of Data: Surface Water, Water Quality, Ground Water

Geographical Coverage: State of Utah

Media in Which Data Stored: Machine readable, Hard copy files, Published reports

Types of Media Cutput: 8 millimeter and 1/4 inch tapes, Computer printout, Published (Printed) reports, Graphics, Floppy disk

MAJOR COMPUTERIZED DATA SYSTEM(S) ACCESSED: NAWDEX, WATSTORE DESCRIPTION OF EQUIPMENT USED: PRIME 9955 Model II minicomputer, Zeta 924/936 Plotter, Data General Aviion Workstations

$* * *$

ORGANIZATION: Earth Science Information Center, U.S. Geological Survey ADDRESS: 222 West 2300 South, 2nd floor, Salt Lake City, UT 84119

TELEPHONE: (801) 975-ESIC Mountain Time

FAX: $975-3740$

NAWDEX CONTACT: Gary Nebeker

AVAILABLE DATA FROM ORGANIZATION:

Types of Data: Surface Water. Water Quality, Ground Water

Geographical Coverage: States of Arizona, Colorado, Idaho, Montana, Nevada,

New Mexico, Utah, Wyoming

Media in Which Data Stored: Hard copy files, Published reports

Types of Media Output: Ad hoc bibliographics

MAJOR COMPUTERIZED DATA SYSTEM(S) ACCESSED: *AGI, Earth Sciences Disc from SilverPlatter.

DESCRIPTION OF EQUIPMENT USED: 386 class IBM PC's

VERMONT

(See U.S. Geological Survey Office in Massachusetts) 


\section{VIRGINIA}

ORGANIZATION: Virginia Water Resources Research Center

ADDRESS: Virginia Polytechnic Institute and State University, 617 North Main Street, Blacksburg, VA 24060

TELEPHONE: (703) 231-8039 Eastern Time

FAX: (703) 231-6673

NAWDEX CONTACT: Diana L. Weigmann

AVAILABLE DATA FROM ORGANIZATION: Streamflow, Climatic, and Peakflow

Types of Data: Surface Water, Precipitation, Meteorological

Geographical Coverage: Nationwide

Media in Which Data Stored: Machine readable, CD-ROM

Types of Media Output: Computer printout, 5.25 or 3.5 inch floppy disk

MAJOR COMPUTERIZED DATA SYSTEM(S) ACCESSED: SWRA

DESCRIPTION OF EQUIPMENT USED: IBM PC/AT and compatibles

ORGANIZATION: U.S. Geological Survey, Water Resources Division

ADDRESS: 3600 West Broad Street, Room 606, Richmond, VA 23230

TELEPHONE: (804) 771-2427

Eastern Time

FAX: (804) 358-0476

NAWDEX CONTACT: Byron J. Prugh, Jr.

AVAILABLE DATA FROM ORGANIZATION:

Types of Data: Surface Water, Water Quality, Ground Water

Geographical Coverage: State of Virginia

Media in Which Data Stored: Machine readable, Hard copy files, Published reports

Types of Media Output: Magnetic tape. Computer printout also 3.5 inch diskette

MAJOR COMPUTERIZED DATA SYSTEM(S) ACCESSED: NAWDEX, WATSTORE DESCRIPTION OF EQUIPMENT USED: PRIME Model 9955

ORGANIZATION: U.S. Geological Survey, Water Resources Division ADDRESS: National Water Data Exchange, 421 National Center, Reston, VA 22092 TELEPHONE: (703) 648-5663 Eastern Time

FAX: (703) 648-57(1)4

NAWDEX CONTACT: Carol Lewis 
AVAILABLE DATA FROM ORGANIZATION: Index information about the availability of surface water, water quality, ground water, meteorological/precipitation data, sediment data, water use data, areal investigations, and other water-resources investigations and related data. Sume data retrieval services are

Geographical Coverage: National available

Media in Which Data Stored: Machine readable, Hard copy files, Microform, Published reports, Ad hoc computer reports

Types of Media Output: Magnetic tape, Computer printout, Microfiche, Published (Printed) reports, Graphics, Statistical analysis, Plots MAJOR COMPUTERIZED DATA SYSTEM(S) ACCESSED: NAWDEX, WATSTORE, STORET, ESIN, NEDRES, ESDD

DESCRIPTION OF EQUIPMENT USED: AMDAHL $470 \mathrm{~V} / 7$ computer and associated hardware, MICRO and PRIME minicomputers, Data General workstations and Personal computers ADDITIONAL COMPUTER SERVICES OR OTHER INFORMATION PRODUCTS PROVIDED BY ORGANIZATION: All NAWDEX documents, direct access by users to the NAWDEX, WATSTORE, and STORET data bases, Data Search Assistance, and specific information products: Water Data Sources Directory, Summary of Indexed Water Data, Catalog and Indexes of Water-Data Acquisition Activities.

ORGANIZATION: Earth Science Information Center, U.S. Geological Survey ADDRESS: $5(17$ National Center, Room 1C4(12, Reston, VA 22092

TELEPHONE: (703) 648-6(1)45 Eastern Time

FAX: (703) 648-5548

NAWDEX CONTACT: Information Services

AVAILABLE DATA FROM ORGANIZATION:

Types of Data: Surface Water, Water Quality, Ground Water

Geographical Coverage: All States

Media in Which Data Stored: Hard copy files, Published reports

Types of Media Output: Computer printout, Ad hoc bibliographics copies of published riports

MAJOR COMPUTERIZED DATA SYSTEM(S) ACCESSED: USGS Library, GNIS, ESDD, DCDB, CC, MCIS, APSRS

DESCRIPTION OF EQUIPMENT USED: Video terminal and letter-quality printer 


\section{WASHINGTON}

ORGANIZATION: U.S. Geological Survey, Water Resources Division

ADDRESS: Suite 600, Pacific Northwest Area, Washington District, 1201 Pacific Avenue, Tacoma, WA 98402

TELEPHONE: (206) 593-6510

Pacific Time

FAX: (206) 593-6514

NAWDEX CONTACT: Luis A. Fuste

AVAILABLE DATA FROM ORGANIZATION:

Types of Data: Surface Water, Water Quality, Ground Water

Geographical Coverage: State of Washington

Media in Which Data Stored: Hard copy files, Published reports

Types of Media Output: Magnetic tape, Floppy disk, Computer printout, Published (Printed) reports

MAJOR COMPUTERIZED DATA SYSTEM(S) ACCESSED: WATSTORE, ADAPS, NWIS DESCRIPTION OF EQUIPMENT USED: PRIME Model 6350

ORGANIZATION: Earth Science Information Center, U.S. Geological Survey ADDRESS: 135 U.S. Post Office, West 904 Riverside Avenue, Spokane, WA 99201 TELEPHONE: (509) 353-2524

Pacific Time

FAX: (509) 353-2872

NAWDEX CONTACT: Thomas L. Servatius

AVAILABLE DATA FROM ORGANIZATION:

Types of Data: Surface Water, Water Quality, Ground Water

Geographical Coverage: States of Alaska (selected), Idaho, Montana, Oregon, Washington

Media in Which Data Stored: Hard copy files, Published reports

Types of Media Output: Ad hoc bibliographics

MAJOR COMPUTERIZED DATA SYSTEM(S) ACCESSED: OCLC CD-ROM, Publications of the USGS CD-ROM, State Water-Data Reports, 1987

Water Year CD-ROM

\section{WEST VIRGINIA}

ORGANIZATION: U.S. Geological Survey, Water Resources Division ADDRESS: $6(13$ Morris Street. Charlestown, WV 25301

TELEPHONE: (304) 347-5130, 5132

Eastern Time

FAX: (304) $347-5133$

NAWDEX CONTACT: Elizabeth Hanna 


\section{WEST VIRGINIA--Continued}

AVAILABLE DATA FROM ORGANIZATION:

Types of Data: Surface Water, Water Quality, Ground Water, Precipitation, Meteorological Water-use

Geographical Coverage: State of West Virginia

Media in Which Data Stored: Machine readable, Hard copy files, Published reports

Types of Media Output: *Magnetic tape (*Shipped from Reston Computer Center), Computer printout, Published (Printed) reports, Diskettes

MAJOR COMPUTERIZED DATA SYSTEM(S) ACCESSED: NAWDEX, WATSTORE, NWIS

DESCRIPTION OF EQUIPMENT USED: PRIME Computer (DMDTWS Towson, MD) Data

General workstations, IBM Personal Computers

ADDITIONAL COMPUTER SERVICES OR OTHER INFORMATION PRODUCTS

PROVIDED BY ORGANIZATION: Programming Assistance, Data Base Technical

Assistance

WISCONSIN

ORGANIZATION: U.S. Geological Survey, Water Resources Division

ADDRESS: 6417 Normandy Lane, Madison, WI 53719

TELEPHONE: (608) 274-3535

Central Time

FAX: (608) 276-3817

NAWDEX CONTACT: Robert Bodoh

AVAILABLE DATA FROM ORGANIZATION:

Types of Data: Surface Water, Water Quality, Ground Water

Geographical Coverage: State of Wisconsin

Media in Which Data Stored: Machine readable, Hard copy files, Published reports

Types of Media Output: Magnetic tape. Computer printout, Published (Printed) reports,

Graphics

MAJOR COMPUTERIZED DATA SYSTEM(S) ACCESSED: NAWDEX, WATSTORE

DESCRIPTION OF EQUIPMENT USED: Calcomp 1(1)39 plotter, PRIME Model 9955,

Data General workstations

WYOMING

ORGANIZATION: Wyoming Water Research Center

ADDRESS: Wyoming University, Post Office Box 3067, University Station, Laramie, WY 82071

TELEPHONE: (307) 766-2143

Mountain Time

FAX: (307) 766-3718

NAWDEX CONTACT: Barry Lawrence 